\title{
The Resistance of Ship Web Girders in Collision and Grounding
}

\author{
Zhenguo Gao, ${ }^{1}$ Song Yang, ${ }^{2}$ and Zhiqiang $\mathrm{Hu}^{1}$ \\ ${ }^{1}$ State Key Laboratory of Ocean Engineering, Shanghai Jiao Tong University, Shanghai 200240, China \\ ${ }^{2}$ China Petroleum Pipeline Engineering Corporation, Langfang 065000, China \\ Correspondence should be addressed to Zhiqiang Hu; zhqhu@sjtu.edu.cn
}

Received 23 June 2014; Accepted 28 July 2014; Published 12 August 2014

Academic Editor: Song Cen

Copyright (C) 2014 Zhenguo Gao et al. This is an open access article distributed under the Creative Commons Attribution License, which permits unrestricted use, distribution, and reproduction in any medium, provided the original work is properly cited.

\begin{abstract}
Ship web girders play an important role in ship structure performance during collision and grounding accidents. The behavior of web girders subjected to in-plane concentrated load is investigated by numerical simulation and theoretical analysis in this paper. A numerical simulation based on previous experiment is conducted to give insight to the deformation mechanism of crushing web girders. Some new important deformation characteristics are observed through the simulation results. A new theoretical deformation model is proposed featured with these deformation characteristics, and a simplified analytical method for predicting the instantaneous and mean resistances of crushing web girders is proposed. The proposed method is verified by two previous experiments and a series of numerical simulations. The agreement between the solutions by the proposed method and the experiment results is good. The comparison results between the proposed analytical method and numerical simulation results are satisfactory for most cases. The proposed analytical method will contribute to the establishment of an efficient method for fast and reliable assessment of the outcome of ship accidental collisions and grounding events.
\end{abstract}

\section{Introduction}

The purpose of present study is to shed light on the deformation behavior of ship web girders subjected to in-plane concentrated load during ship collision and grounding accidents. By numerical simulation and plastic mechanism analysis, the deformation mode and associated energy dissipation are investigated. Furthermore, the resistance of web girders can be obtained by the proposed simplified analytical method, which can be a useful tool in quick and reliable assessment of the ship structures during accidental situations.

Although many efforts such as the application of advanced navigation tools have been made to enhance the safety level of sailing ships, the risk of ship collision and grounding still does exist. Such accidents can lead to serious consequences including environmental pollution, casualties, and economic loss. For example, the collision between Atlantic Empress and Aegean Captain in 1979 resulted in the pouring of approximately 90 million gallons of oil into a pristine wilderness area near Tobago. The grounding accident of Costa Concordia cruise ship near the shore of Italy led to the perishing of 32 lives.
As more attention has been paid to the catastrophic consequences caused by such disasters, great efforts have been put into understanding of the internal mechanics of ship structures subjected to collision and grounding in the past several decades. The existing approaches are prevailingly divided into three categories, experimental method, nonlinear finite element method (NLFEM), and simplified analytical method. Experimental method can be considered as the most convincing approach, but full scale experiments are too risky and expensive to conduct, and small scale experiments are difficult to be interpreted to full scale because of complicated scaling effects. NLFEM, which is considered as "numerical experiment," has exhibited great abilities for simulating certain large scale ship collision and grounding accidents benefitting from the rapid progress of computer technology. As long as the modeling parameters are properly set, NLFEM can provide significant details and satisfactory results to the deformation process of structures subjected to accidental loads. Moreover, NLFEM has the advantage of low costs and repeatable analyses. Therefore, NLFEM has been extensively used in the ship structure analysis. For example, Haris and Amdahl [1] used the FE software 


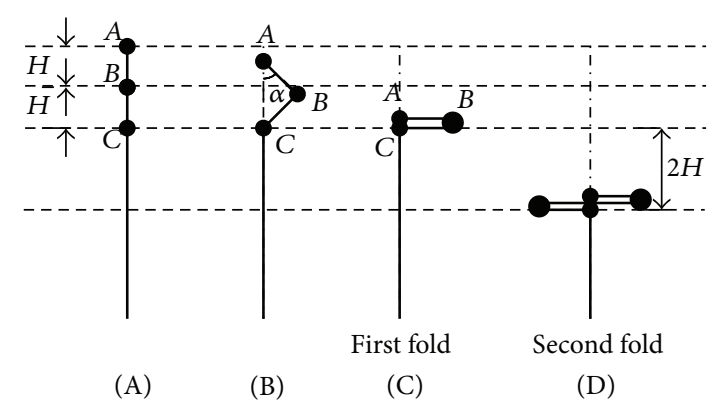

(a) Model I

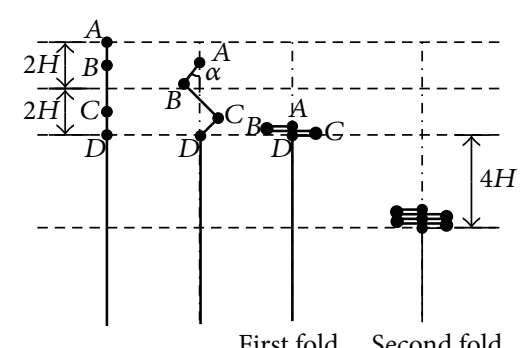

(A)
(B) (C)

(b) Model II

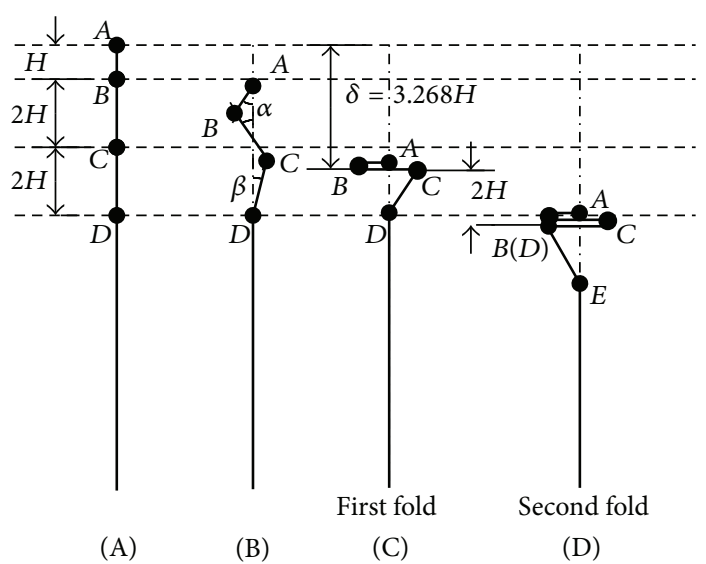

(c) Model III

Figure 1: Progressive folding of the cross section in the loading plane of various denting modes for web girders (the filled small circles represent plastic hinges) [17].

LS_DYNA to produce virtual experimental data for several ship collision scenarios and used the numerical results to validate a simplified analytical method for ship collision. Yu et al. [2] and Zhiqiang et al. [3] conducted numerical simulations to verify a simplified analytical method for the predictions of structural performance during ship grounding over seabed obstacles with large contact surfaces. Kitamura [4], Endo et al. [5], and Yamada and Endo [6] analyzed a series of buffer-bow designs with numerical simulations. Alsos et al. [7] investigated the failure criterions with respect to fracture with numerical analyses.

Compared with above-mentioned two methods, simplified analytical method shows its advantages of simplicity and reasonable accuracy since the method is based on the plastic mechanism analysis originating from the upper bound theorem. The method has been extensively used by many researchers, for example, Amdahl [8], Wang [9], Simonsen [10], and Hong [11], for estimating the collapse load of ship structure subjected to extreme loads. It can be found that the estimating results always show good agreement with experiments. This is mainly due to the fact that the simplified analytical method can provide significant sight into the governing physical process to the deformation of ship structures. Thus, simplified analytical method is especially suitable for the situations when quick evaluation of the ship structure performance is essential, such as in the preliminary design stage and emergency situation when decision supporting tools for crisis handling are needed.

Ship web girders, including transverse frame, stringer, deck plating, primary bulkhead, longitudinal girder, and bottom floor, play important roles in ship crashworthiness, as they can absorb a large portion of striking energy when accidents occur. In many situations, ship web girders suffer in-plane loads; for example, the web frame or stringer may suffer in-plane impact force in a collision process and the floor may be crushed by rock during stranding.

The deformation of web girders subjected to in-plane concentrated loads has been studied by many researchers with experiments and NLFEM. A variety of simplified analytical methods for predicting the crushing resistance of web girders have also been proposed based on plastic mechanism analysis of theoretical deformation models. Wierzbicki and Culbertson Driscoll [12] pioneered to propose a simplified model for the postbucking deformation and verified it by small-scale crushing tests of extruded aluminum doublehollow forms. Wang [9], Simonsen [13], and Zhang [14] proposed various formulae for predicting crushing resistance of web girders subjected to in-plane localized load based on the same theoretical deformation model but with different presuppositions; see Model I in Figure 1(a). Simonsen and Ocakli [15] conducted a series of experimental analysis of deep plastic collapse of web girders subjected to an in-plane 
TABLE 1: Summary of some existing simplified analytical methods for predicting the crushing resistance of web girders.

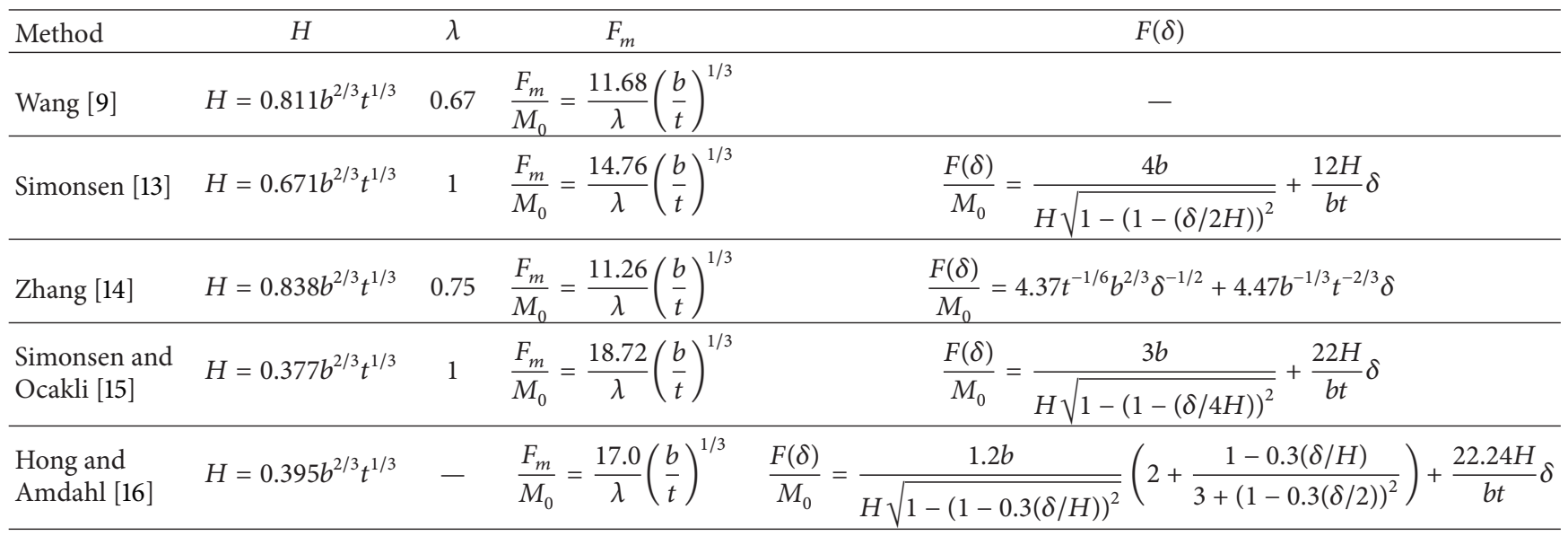

concentrated load. Based on some captured new deformation features during the crushing process of web girders in the experiments, they developed a new theoretical deformation model, as shown in Figure 1(b), and, furthermore, a new simplified analytical method for crushing web girders was obtained. Hong and Amdahl [16] conducted a comparative study of the existing simplified analytical methods and investigated the different formulae in terms of instantaneous and mean crushing resistance. Then a more complex theoretical deformation model was proposed based on the numerical simulation, as shown in Figure 1(c). The formulae for predicting the instantaneous resistance and mean resistances of crushing web girders were derived based on the plastic mechanism analysis of the model. The existing simplified analytical methods for predicting crushing resistance of web girders that suffer in-plane concentrate load are summarized in Table 1.

In the present analysis, numerical simulation of crushing web girders based on a previous model test is conducted. Some important deformation features during the crushing process, which have been ignored by the existing theoretical deformation models, are captured through a detailed analysis of the numerical results. Based on the new found features, a new theoretical deformation model is proposed for crushing web girders subjected to in-plane localized load. Then the energy dissipation of the new theoretical model is identified and the analytical formulae for both the instantaneous resistance and mean resistance of crushing web girders are built. Furthermore, the proposed simplified analytical method is verified by experiments and numerical simulations. It can be found that the prediction results by the new method agree satisfactorily with the results of experiments and numerical simulation cases.

\section{Numerical Simulation}

In the present study, a numerical simulation of crushing web girder is conducted with NLFEM software firstly, on purpose of examining the structural deformation mode of web girder. The crushing scenario is chosen as the ASIS model test [18]
TABLE 2: Scantling of the model in ASIS test [18].

\begin{tabular}{lcc}
\hline Deck height & $H_{w}$ & $1635 \mathrm{~mm}$ \\
Overall length & $L$ & $6000 \mathrm{~mm}$ \\
Web spacing & $2 b$ & $2000 \mathrm{~mm}$ \\
Width of side plating & $2 a$ & $450 \mathrm{~mm}$ \\
Deck plate thickness & $t$ & $7 \mathrm{~mm}$ \\
Side plating thickness & $t_{f}$ & $10 \mathrm{~mm}$ \\
Stiffener thickness & $t_{s}$ & $8 \mathrm{~mm}$ \\
\hline
\end{tabular}

case and all the FE models in the numerical simulations are made based on ASIS model test [18]. The ASIS model test [18] was carried out in 1993 to study the failure mechanism and energy absorption capacity of a tanker's side structures. The test scale was set as $1: 2$. The model was a part of the side structures of a VLCC with one space of longitudinal stiffeners and three spaces of transverse webs included, as Figure 2(a) shows. The indenter model in ASIS test is shown in Figure 2(b). The collision position was set between two adjacent transverse webs and in the plane of the deck plate. Material of the models was mild steel for structures. In the ASIS test, both dynamic and static experiments were carried out. The deformation of the model after static test is shown in Figure 3. Here only static experiment scenario is performed in the numerical simulation. The main data of the model test are listed in Table 2.

The FE model of side structure in numerical simulation is modeled with code Patran 2012, including side shell, deck plate, transverse webs, and attached stiffeners with the scantling in Table 2. The indenter is modeled with length $1.0 \mathrm{~m}$ and height $1.0 \mathrm{~m}$; the lower part has a radius of $0.5 \mathrm{~m}$. The FE models in crushing simulation are shown in Figure 4. The commercial code LS-DYNA version 971 is used to calculate structural performance during crushing process.

For the side structure FE model, the element choice is four nodes quadrilateral Belytschko-Tsay (ELFORM2) [19]. Due to the simplicity of the model, the fine mesh size $20 \mathrm{~mm}$ is chosen for the whole side structure to give satisfactory accuracy and the contact part between the indenter and the 


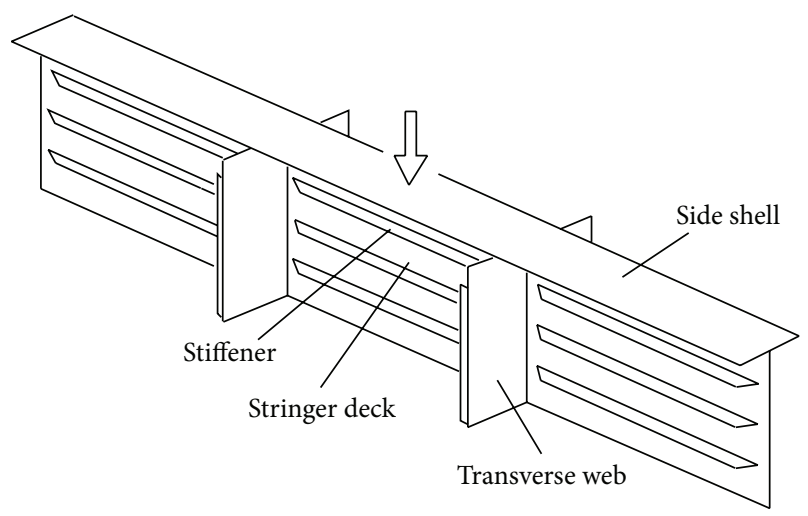

(a)

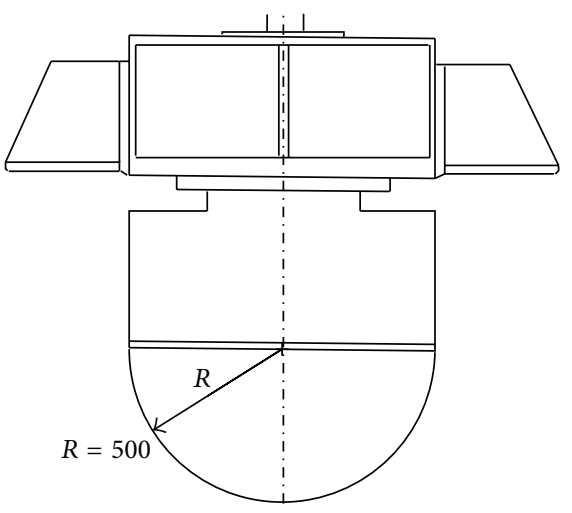

(b)

FIGURE 2: The models in ASIS test: (a) ship side structure; (b) the indenter [18].

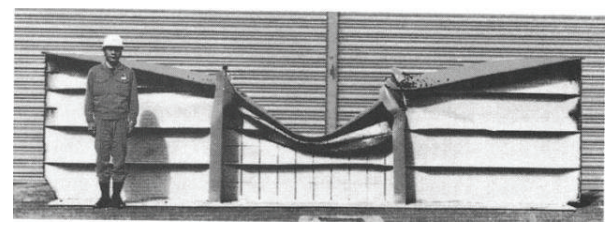

Figure 3: ASIS static model test.

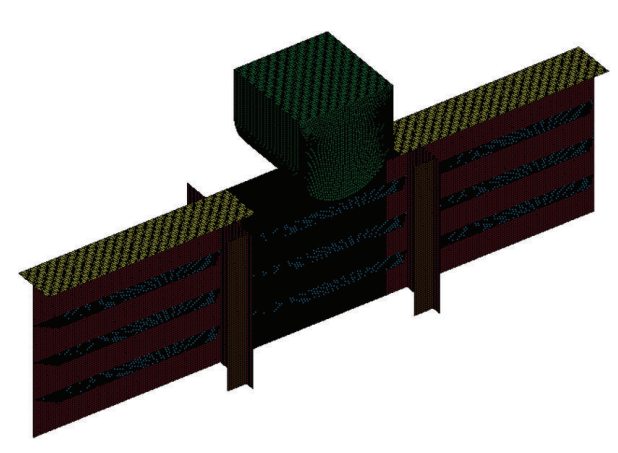

FIGURE 4: The FE models in numerical simulation.

side structure is meshed with a more detailed mesh size of $10 \mathrm{~mm}$. An elastic-plastic material with a yield stress of $310 \mathrm{MPa}$ and a relatively small piecewise linear hardening is used for the side structure model. The side structure model is restricted at longitudinal ends by fixing the six degrees of freedom. The same restriction is also applied to the inner edge of the deck plate. The indenter is assumed to be rigid. A crushing speed of $2 \mathrm{~m} / \mathrm{s}$ for indenter is adopted, enabling a good balance between calculation accuracy and computational cost as crushing speed has slight influence on the crushing behavior [20].

The automatic surface to surface contact of LS_DYNA is employed to treat the contact between the indenter and side structures, while the contacts between structural components of side structures are treated with the automatic single surface contact of LS_DYNA. The friction coefficient between the indenter and side structures is set to be 0.3 and the same value is employed in the internal contact of crushed side structures.

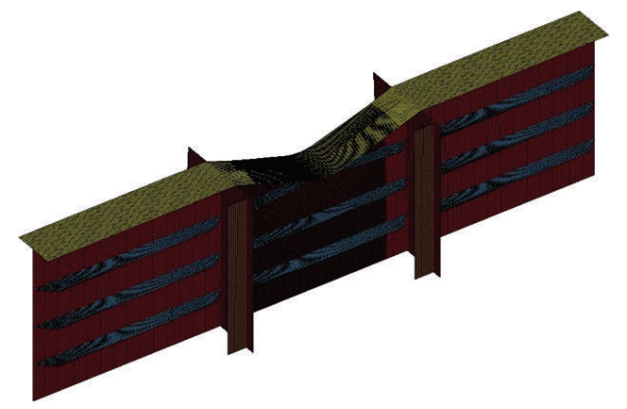

FIGURE 5: Deformation of side structures in numerical simulation.

The deformation of the side structures can be observed in Figure 5, and the indenter is removed for the ease of observation. The result of the numerical simulation is compared with that of ASIS model test in terms of instantaneous forceindentation curve; see Figure 6 . It can be found that the forceindentation curve obtained from numerical simulation shows perfect agreement with the ASIS model test data, which means the numerical simulation produces a good representation of ASIS model test. Thus, the deformation process derived from the postprocessing of the numerical simulation is reliable to give insight into deformation mechanism of web girders subjected to in-plane concentrated load, especially before rupture occurs.

The progressive crushing deformation process of middle cross section of the deck plate is shown in Figure 7. It can be found that the plate bulges out of the original plane of the girder and folds to both sides in turn. During the first folding process, the uppermost wrinkle of the plate is shorter than the middle part, which is captured by most of the existing theoretical deformation models. However, the existing simplified analytical methods usually assume a depth scale of $1: 2$ for the upper two wrinkles of the first fold, while a depth scale of nearly $1: 4$ is observed here. Based on this, a new theoretical deformation model is proposed incorporating the above observations. 


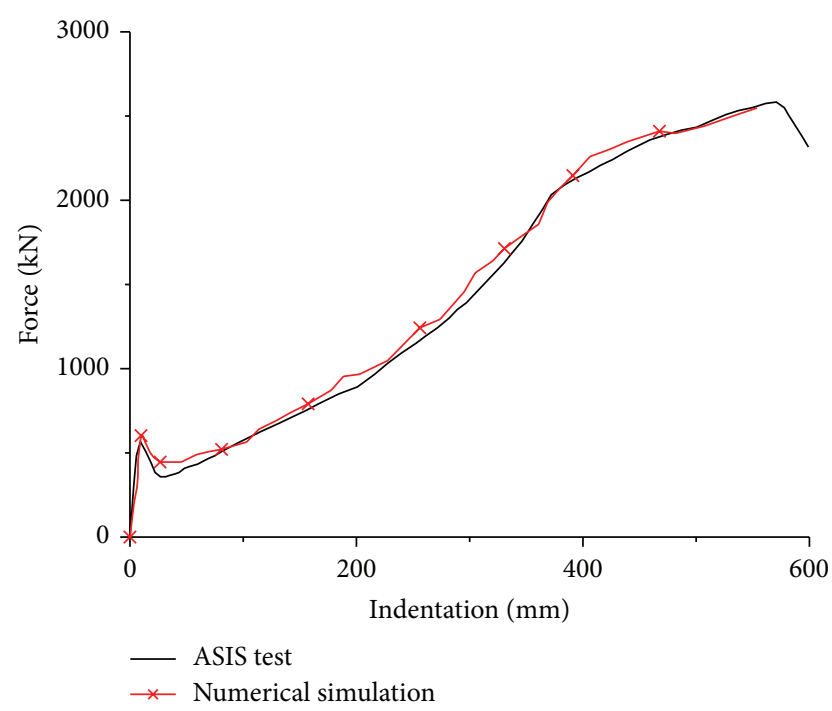

FIgURE 6: Comparisons of the numerical simulation with ASIS model test.

\section{A New Simplified Analytical Method for Crushing Web Girder}

A new theoretical deformation model for crushing web girder is proposed based on the previous analysis, as shown in Figure 8. The initial bulking, as the first peak of the curves in Figure 6 shows, is neglected and instead the final shape of compressed fold is used to identify the idealized deformation model. The web girder is assumed to be crushed eccentrically for generality and only one side with length $b_{1}$ is shown in Figure 8 , while the other side with length $b_{2}=b-b_{1}$ can be treated in the same way. The folding process of the crushing web girder cross section in the loading plane is shown in Figure 9 for a better understanding of the new theoretical deformation model.

For the first fold in the new model, the uppermost wrinkle is a quarter of the depth of the middle wrinkle, which means $B C=4 A B=4 H$ in Figures 8 and 9. The third wrinkle of the first fold is set to be $3 \mathrm{H}$ for the geometric continuity. Thus, the first fold is completely flattened when the crushing indentation is $8 H$, which means that the characterized depth of the first fold is $8 \mathrm{H}$. As indentation increases, more subsequent folds will form and fold to both sides equally in turn. To accommodate the first fold, $6 \mathrm{H}$ is the characterized depth of the subsequent folds since the extension of the subsequent folds does not exceed that of the first fold.

Based on the new model, the instantaneous and mean crushing resistances of the web girder will be obtained through plastic mechanism analysis.

According to the upper bound theorem, the instantaneous force can be derived by equating the rate of work for external load with the rate of internal energy dissipation. The equilibrium can be expressed as

$$
F(\delta) \cdot \dot{\delta}=\dot{E}_{\text {int }}
$$

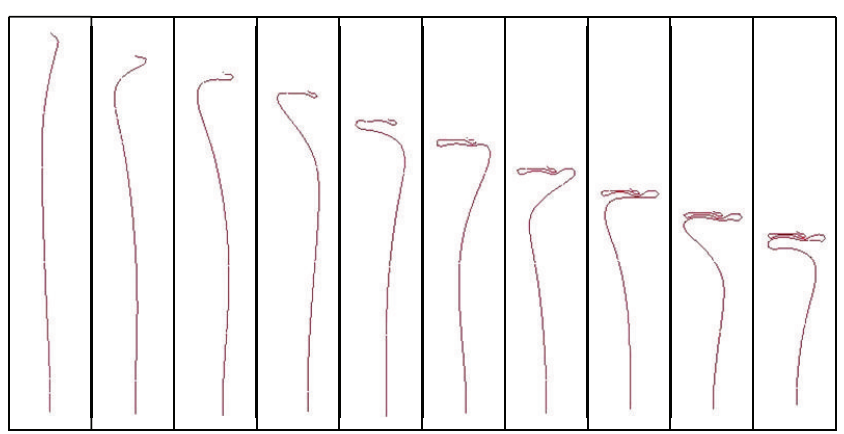

FIGURE 7: Crushing deformation process of the middle cross section of the deck plate.

where $F(\delta)$ is the instantaneous resistance of the web girder, which equals the external crushing load, $\dot{\delta}$ is the crushing velocity, and $\dot{E}_{\text {int }}$ is the internal strain energy dissipation rate, which can be separated into bending energy dissipation rate $\dot{E}_{b}$ and membrane energy dissipation rate $\dot{E}_{m}$; that is,

$$
\dot{E}_{\text {int }}=\dot{E}_{b}+\dot{E}_{m}
$$

It is assumed that two energy dissipation patterns are decoupled from each other, and bending energy dissipation is concentrated on plastic hinge lines. The material is assumed to be rigid perfectly plastic, and the effect of elasticity is neglected. A flow stress $\sigma_{0}$, which is taken as the average value of the yield stress $\sigma_{y}$ and ultimate stress $\sigma_{u}$, is chosen in order to take hardening effect into consideration.

3.1. Plastic Mechanism Analysis of the First Fold. During the first folding process of the new model, the bending energy dissipation concentrates in the eight plastic hinge lines. Based on the assumption that $8 H \ll\left(b_{1}, b_{2}\right)$, the rate of bending energy dissipation can be expressed as

$$
\dot{E}_{b}=6 M_{0}\left(b_{1}+b_{2}\right) \dot{\alpha}
$$

where $\dot{\alpha}$ is angular bending rate and $M_{0}$ is the fully plastic bending moment capacity of a plate strip with unit width and plate thickness $t$, which is given by

$$
M_{0}=\frac{\sigma_{0} t^{2}}{4} \text {. }
$$

The instantaneous indentation $\delta$ and instantaneous rotation angle $\alpha$, as shown in Figure 9, can be related by

$$
\delta=8 H(1-\cos \alpha)
$$

where $H$ is the height of the upper wrinkle in the first fold and is a free parameter.

The angular bending rate $\dot{\alpha}$ can be related to crushing velocity $\dot{\delta}$ as

$$
\dot{\alpha}=\frac{\dot{\delta}}{8 H \sqrt{1-(1-\delta / 8 H)^{2}}} .
$$




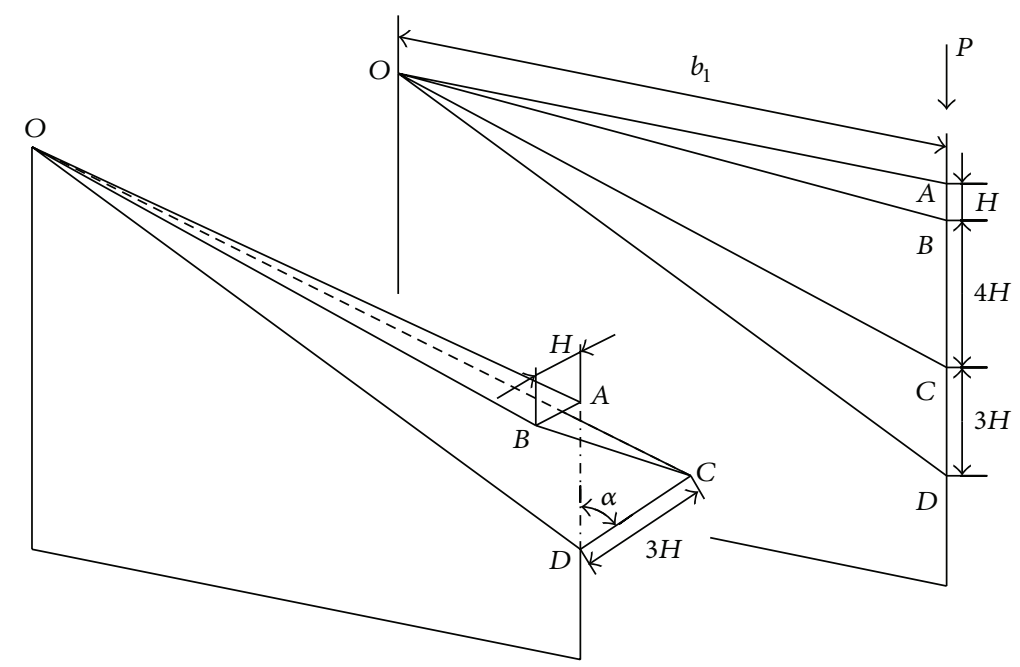

FIgURE 8: New deformation model of the web girder (one side with length $b_{1}$ ).

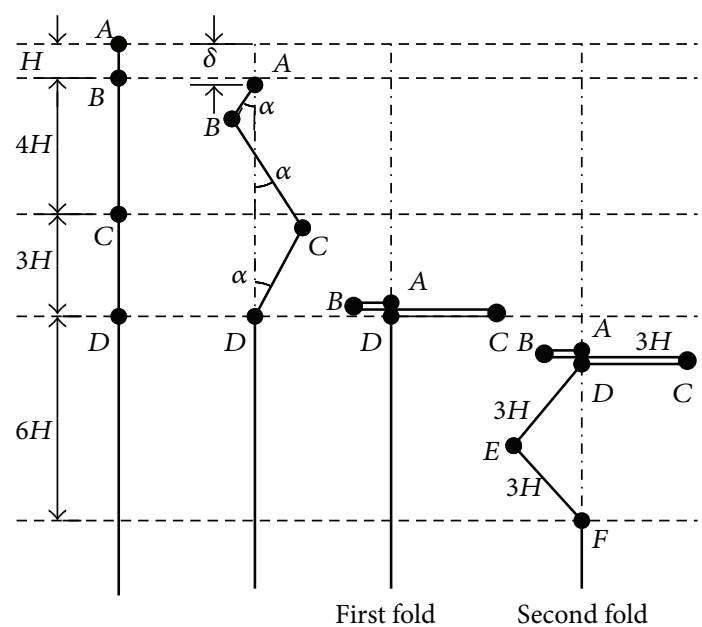

FIGURE 9: Folding process of the crushing girder cross section in the loading plane (the black dots represent plastic hinges).

When the first fold is completely flattened, the instantaneous rotation angle $\alpha$ increases from 0 to $\pi / 2$; the total bending energy dissipation can be integrated as

$$
E_{b}=3 \pi M_{0}\left(b_{1}+b_{2}\right) .
$$

For the membrane energy dissipation in the first fold, Simonsen and Ocakli [15] found that the minimization of the energy dissipation can be achieved when the membrane energy is only dissipated by direct tensile straining, which means that only the strain of material fibers parallel to the outer plate edge is considered. Thus, the membrane energy dissipation can be obtained simply by

$$
\dot{E}_{m}=\int_{A} N_{0} \dot{\varepsilon}_{\mathrm{avg}} d A
$$

where $A$ is the deforming area of the web girder, $\dot{\varepsilon}_{\text {avg }}$ is the average tensile strain rate in the length direction of the plate, and $N_{0}$ is the fully plastic membrane force per unit length of the plate and can be formulated as

$$
N_{0}=\sigma_{0} t
$$

During the first folding process, the indentation $\delta$ increases from 0 to $8 H$, and the mean strain of the uppermost fiber is

$$
\varepsilon_{\mathrm{OA}}=\frac{1}{2}\left(\frac{\delta}{b_{1}}\right)^{2} .
$$

Thus, the strain rate of the uppermost fiber can be expressed as

$$
\dot{\varepsilon}_{\mathrm{OA}}=\frac{\delta}{b_{1}^{2}} \dot{\delta} .
$$

From the consideration of geometry, the strain rate of other hinge lines can be formulated as

$$
\begin{gathered}
\dot{\varepsilon}_{\mathrm{OB}}=\dot{\varepsilon}_{\mathrm{OA}}=\frac{\delta}{b_{1}^{2}} \dot{\delta}, \\
\dot{\varepsilon}_{\mathrm{OC}}=0.6 \frac{\delta}{b_{1}^{2}} \dot{\delta}, \\
\dot{\varepsilon}_{\mathrm{OD}}=0 .
\end{gathered}
$$

The average tensile strain rate in the length direction over the entire height during the first folding process can be expressed as

$$
\dot{\varepsilon}_{\text {avg }}=0.6375 \frac{\delta}{b_{1}^{2}} \dot{\delta} .
$$

Applying (8) to two sides of the web girder with lengths $b_{1}$ and $b_{2}$, the membrane energy dissipation rate is

$$
\dot{E}_{m}=5.1 N_{0} H\left(\frac{1}{b_{1}}+\frac{1}{b_{2}}\right) \delta \dot{\delta} .
$$


Integrating (14) over the entire first folding process, that is, $\delta$ increases from 0 to $8 H$, the total membrane energy dissipation is obtained as

$$
E_{m}=163.2 N_{0} H^{3}\left(\frac{1}{b_{1}}+\frac{1}{b_{2}}\right) .
$$

When the bending energy dissipation rate and membrane energy dissipation rate are both obtained, the instantaneous resistance of the web girder can be derived by (1) and (2):

$$
\begin{aligned}
F(\delta)= & \frac{\dot{E}_{b}+\dot{E}_{m}}{\dot{\delta}}=\frac{0.75 M_{0}\left(b_{1}+b_{2}\right)}{H \sqrt{1-(1-\delta / 8 H)^{2}}} \\
& +5.1 N_{0} H \delta\left(\frac{1}{b_{1}}+\frac{1}{b_{2}}\right),
\end{aligned}
$$

where $0 \leq \delta \leq 8 H$.

The mean resistance of a crushing web girder during the formation of a fold can be derived by dividing total energy by the crushing indentation. The mean resistance can be expressed as

$$
\begin{aligned}
F_{m}= & \frac{E_{b}+E_{m}}{8 H}=\frac{0.375 \pi M_{0}\left(b_{1}+b_{2}\right)}{H} \\
& +20.4 N_{0} H^{2}\left(\frac{1}{b_{1}}+\frac{1}{b_{2}}\right) .
\end{aligned}
$$

Up to now, $H$ is still a free parameter and is chosen here to be the value which minimizes the mean force and also the total energy dissipation in line with upper-bound methods for infinitesimal deformations. That is,

$$
\frac{\partial F_{m}}{\partial H}=0
$$

Substituting (17) into (18), $H$ can be obtained as

$$
H=0.193\left(b_{1} b_{2} t\right)^{1 / 3} .
$$

Introducing the value of $H$ to (16) and (17), the instantaneous and mean forces of crushing web girder for the first fold can be calculated easily.

When the web girder is crushed centrically, that is, $b_{1}=$ $b_{2}=b$, the $H$ and the instantaneous and mean resistances are expressed as follows:

$$
\begin{aligned}
H & =0.193 b^{2 / 3} t^{1 / 3} \\
F(\delta) & =\frac{1.5 M_{0} b}{H \sqrt{1-(1-\delta / 8 H)^{2}}}+\frac{10.2 N_{0} H \delta}{b}, \\
F_{m} & =\frac{0.75 \pi M_{0} b}{H}+\frac{40.8 N_{0} H^{3}}{b},
\end{aligned}
$$

where $0 \leq \delta \leq 8 H$.

In the existing simplified analytical methods for web girders, many researchers use the effective crushing factor in their formulae. This is due to the issue of effective crushing distance, which is studied by researchers such as Amdahl [8] and Abramowicz [21]. It can be found that the web girder plate cannot be compressed entirely during the folding process. The actual crushing distance is usually smaller than the theoretical crushing distance because of the plate thickness and the radius formed in the plastic hinge lines. Therefore, a dimensionless effective crushing factor $\lambda$ is introduced by dividing the actual crushing distance by the theoretical crushing distance. The values of the effective crushing factor employed in the existing simplified analytical methods are shown in Table 1. In the present analysis, the effective crushing factor $\lambda$ is assumed to be 1.0 .

3.2. Subsequent Folding. After the first fold is completely flattened, and if fracture of the web girder does not happen, the crushing process will continue with the mechanism described in Figure 9. The subsequent folds will form as the indentation increases. As this occurs in most realistic cases, there is a need to predict the resistance during subsequent folding by extending the capability of the present theory.

As shown in Figure 9, the subsequent folds will be characterized by $6 \mathrm{H}$ and equally fold to both sides in turn. The characteristic crushing wavelength $H$ expressed by (19) is still valid for subsequent folding. Similarly with the first fold, the energy dissipation of the subsequent folds can be separated into bending energy dissipation and membrane energy dissipation.

During the second folding process, the flattened first fold still has the capacity to dissipate membrane energy. Thus, the second term in (16) must be kept in the instantaneous resistance calculation of the second fold. The instantaneous resistance contributed from the total membrane energy dissipation during the second folding process can be expressed as

$$
\begin{aligned}
F(\delta)_{\text {membrane }}= & 5.1 N_{0} H \delta\left(\frac{1}{b_{1}}+\frac{1}{b_{2}}\right) \\
& +5.1 N_{0} H(\delta-8 H)\left(\frac{1}{b_{1}}+\frac{1}{b_{2}}\right) \\
= & 10.2 N_{0} H(\delta-4 H)\left(\frac{1}{b_{1}}+\frac{1}{b_{2}}\right),
\end{aligned}
$$

where $8 H<\delta \leq 14 H$

The instantaneous resistance due to the bending energy dissipation is approximated from smearing the total bending energy dissipation over the indentation depth of the second fold. It can be formulated as

$$
F(\delta)_{\text {bending }}=\frac{\pi M_{0}\left(b_{1}+b_{2}\right)}{3 H} .
$$

The instantaneous resistance of the web girder during the second folding process is obtained by summation of (21) and (22):

$$
\begin{aligned}
F(\delta) & =F(\delta)_{\text {membrane }}+F(\delta)_{\text {bending }} \\
& =10.2 N_{0} H(\delta-4 H)\left(\frac{1}{b_{1}}+\frac{1}{b_{2}}\right)+\frac{\pi M_{0}\left(b_{1}+b_{2}\right)}{3 H} .
\end{aligned}
$$


Similarly, the crushing resistance of the third fold, the forth fold, and so forth, can be determined. For the $n$th $(n>1)$ fold, the instantaneous resistance due to the membrane energy dissipation can be expressed as

$$
\begin{aligned}
F(\delta)_{\text {membrane }}= & 5.1 N_{0} H \delta\left(\frac{1}{b_{1}}+\frac{1}{b_{2}}\right) \\
& +5.1 N_{0} H(\delta-8 H)\left(\frac{1}{b_{1}}+\frac{1}{b_{2}}\right) \\
& +5.1 N_{0} H(\delta-14 H)\left(\frac{1}{b_{1}}+\frac{1}{b_{2}}\right)+\cdots \\
& +5.1 N_{0} H(\delta-2(3 n-2) H)\left(\frac{1}{b_{1}}+\frac{1}{b_{2}}\right) \\
= & 5.1 N_{0} H(n \delta-(n-1)(3 n-2) H)\left(\frac{1}{b_{1}}+\frac{1}{b_{2}}\right) .
\end{aligned}
$$

The instantaneous resistance due to the bending energy dissipation for the $n$th $(n>1)$ fold can still be formulated as (22). Thus, the crushing resistance of the web girder upon the $n$th $(n>1)$ fold can be expressed as

$$
\begin{aligned}
F(\delta)= & F(\delta)_{\text {membrane }}+F(\delta)_{\text {bending }} \\
= & 5.1 N_{0} H(n \delta-(n-1)(3 n+2) H)\left(\frac{1}{b_{1}}+\frac{1}{b_{2}}\right) \\
& +\frac{\pi M_{0}\left(b_{1}+b_{2}\right)}{3 H} .
\end{aligned}
$$

The web girder is usually mounted to a plate or fitted with a flange. To compare with the previous experiments, it is necessary to include the resistance of the attached flange plate. In the present study, the resistance of the flange plate is estimated by a beam model, which is also adopted by Hong and Amdahl [16]. The resistance-indentation relation of the flange plate can be expressed as

$$
F_{f}(\delta)=\frac{4 \sigma_{0} a t_{f}}{b} \delta
$$

where $F_{f}(\delta)$ is the resistance of the flange plate, $a$ is the half width of the plate, $b$ is the half span of the plate, $t_{f}$ is the plate thickness, $\sigma_{0}$ is the flow stress, and $\delta$ is the indentation.

Based on the assumption that interaction between different structural elements is neglected, the total instantaneous resistance of the web girder with attached flange can be obtained by the summation of instantaneous resistances of web girder and flange. That is,

$$
F_{w}(\delta)=F(\delta)+F_{f}(\delta)
$$

where $F_{w}(\delta)$ is the total instantaneous resistance of the web girder with attached flange.
TABLE 3: Scantling of the model in the experiment by Simonsen and Ocakli [15].

\begin{tabular}{lcc}
\hline Flow stress & $\sigma_{0}$ & $250 \mathrm{MPa}$ \\
Depth of web girder & $H_{w}$ & $150 \mathrm{~mm}$ \\
Span of web girder & $2 b$ & $150 \mathrm{~mm}$ \\
Width of web flange & $2 a$ & $50 \mathrm{~mm}$ \\
Plate thickness of web girder & $t$ & $1 \mathrm{~mm}$ \\
Plate thickness of web flange & $t_{f}$ & $1 \mathrm{~mm}$ \\
\hline
\end{tabular}

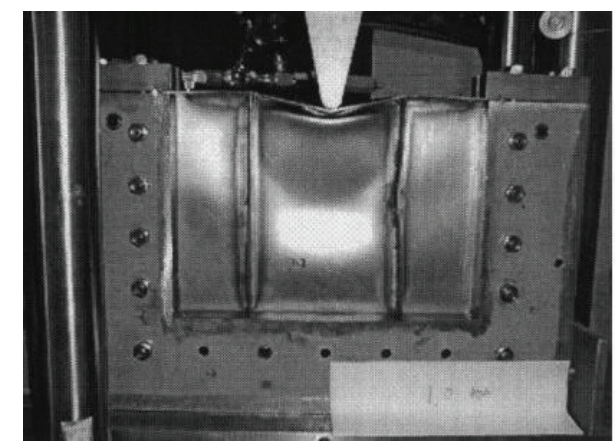

FIgure 10: The model test by Simonsen and Ocakli [15].

\section{Verification}

4.1. Verification by Experiments. The proposed simplified analytical method for predicting resistance of crushing web girders is verified by two previous experiments, which have been widely used in the verifications of the plastic analytical methods in the ship structure crashworthiness research.

Simonsen and Ocakli [15] carried out a series of experiments on web girder crushing in DTU in 1999. The scale of the experiments was 1:20. Zhang [14] and Simonsen and Ocakli [15] both used the experiments to verify their formulae, respectively. One of the experiments is adopted here to verify the proposed simplified analytical method. The scantling of the model in the experiment is listed in Table 3. The experiment scenario is shown in Figure 10.

The other experiment employed for verifying the proposed simplified analytical method is ASIS model test, which has been introduced in Section 2.

The comparisons between the simplified analytical method and the experiments are shown in Figure 11. The force-indentation curves calculated by other existing simplified analytical methods are also plotted in Figure 11.

4.2. Verification by Numerical Simulations. In the present study, a series of numerical simulations are conducted to verify the proposed simplified analytical method. Eleven cases with three kinds of plate length-thickness $(2 b / t)$ ratio for web girders are defined in the numerical simulations. The numerical simulations cover a wide range of scales, and the length-depth ratio of the web girder is set to be $2: 1$ in all cases. The scantlings of the web girders and attached flange plates in eleven cases are listed in Table 4.

Stiffeners are not considered here. Hong and Amdahl [16] investigated the effect of stiffeners on crushing resistance of 


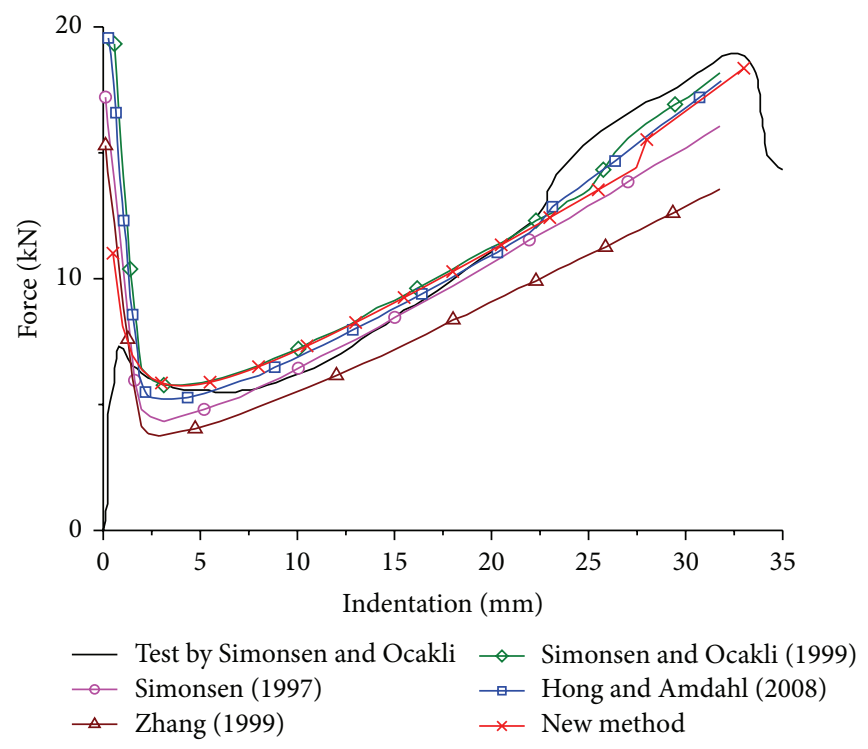

(a)

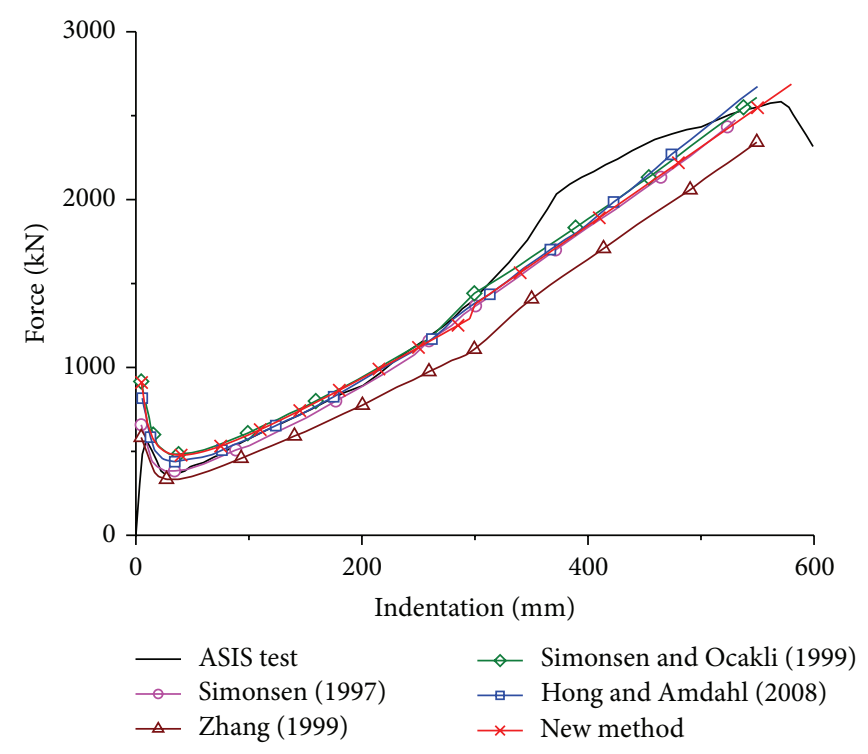

(b)

FIGURE 11: Comparisons of the new method with the experiments: (a) with test by Simonsen and Ocakli; (b) with ASIS test.

TABLE 4: Scantlings of web girders and flange plates in numerical simulations.

\begin{tabular}{|c|c|c|c|c|c|c|c|}
\hline \multirow{2}{*}{$2 b / t$} & \multirow{2}{*}{ Case no. } & \multicolumn{3}{|c|}{ Web girder plate $(\mathrm{mm})$} & \multicolumn{3}{|c|}{ Flange plate $(\mathrm{mm})$} \\
\hline & & Length & Height & Thickness & Length & Width & Thickness \\
\hline \multirow{3}{*}{100} & $\mathrm{Cl}$ & 400 & 200 & 4 & 400 & 100 & 5 \\
\hline & $\mathrm{C} 2$ & 800 & 400 & 8 & 800 & 200 & 10 \\
\hline & $\mathrm{C} 3$ & 1600 & 800 & 16 & 1600 & 200 & 18 \\
\hline \multirow{4}{*}{200} & $\mathrm{C} 4$ & 400 & 200 & 2 & 400 & 100 & 2 \\
\hline & $\mathrm{C} 5$ & 800 & 400 & 4 & 800 & 200 & 5 \\
\hline & $\mathrm{C} 6$ & 1600 & 800 & 8 & 1600 & 200 & 10 \\
\hline & $\mathrm{C} 7$ & 3200 & 1600 & 16 & 3200 & 200 & 18 \\
\hline \multirow{4}{*}{400} & $\mathrm{C} 8$ & 400 & 200 & 1 & 400 & 100 & 2 \\
\hline & C9 & 800 & 400 & 2 & 800 & 200 & 2 \\
\hline & $\mathrm{C} 10$ & 1600 & 800 & 4 & 1600 & 200 & 5 \\
\hline & $\mathrm{C} 11$ & 3200 & 1600 & 8 & 3200 & 200 & 10 \\
\hline
\end{tabular}

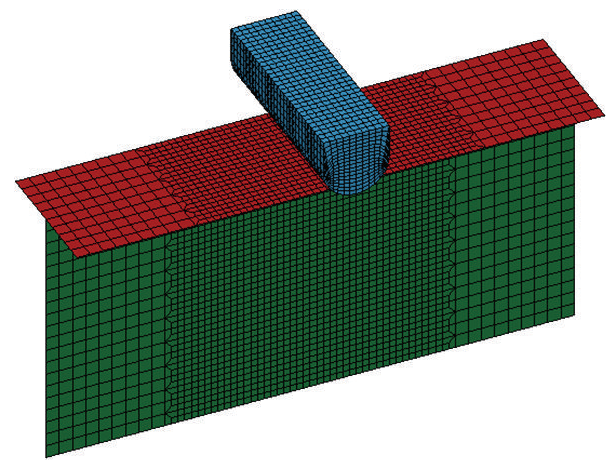

FIGURE 12: FE models in numerical simulation.

web girders, and it was found that web girders with different longitudinal stiffener spacing exhibit similar crushing resistance. The effect of longitudinal stiffeners is small and only closely spaced stiffeners will increase the crushing resistance of web girders to some certain degree. For most realistic cases, the effect of stiffeners can be considered by smearing the cross-sectional area of stiffeners into the plate thickness to assess the resistance of the plate; this has been widely accepted.

In all cases, the indenter is assumed to be rigid, with length $300 \mathrm{~mm}$ and height $100 \mathrm{~mm}$, and the radius of the lower part is $50 \mathrm{~mm}$. The crushing speed of the indenter is $800 \mathrm{~mm} / \mathrm{s}$. The other settings and parameters are all the same as those in the previous numerical simulation. The FE models in the numerical simulations are shown in Figure 12.

The force-indentation curves in all cases are obtained after postprocess of the numerical simulations. The comparisons between the numerical simulations and new simplified analytical method are shown in Figure 13.

4.3. Discussion. From the comparisons between the new method and experiments shown in Figure 11, it can be found 


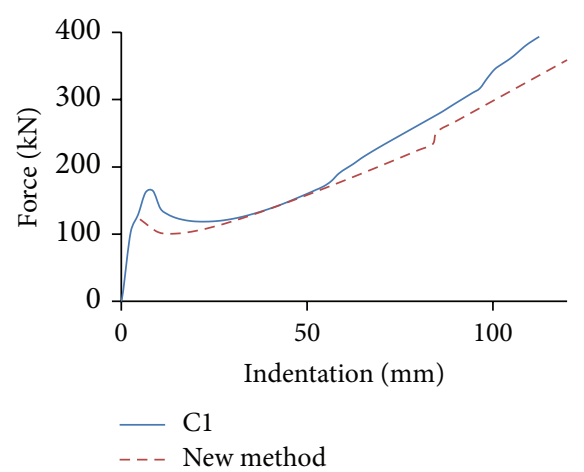

(a)

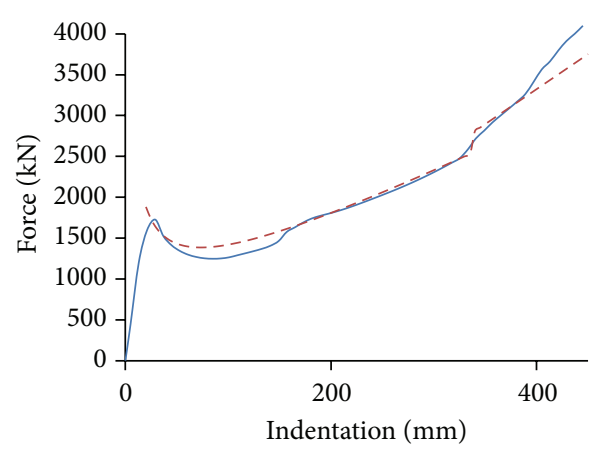

$-\mathrm{C} 3$

- _ - New method

(c)

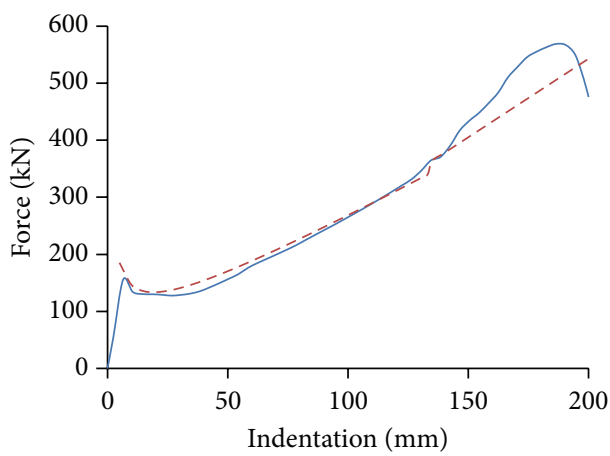

$-\mathrm{C} 5$

- _ - New method

(e)

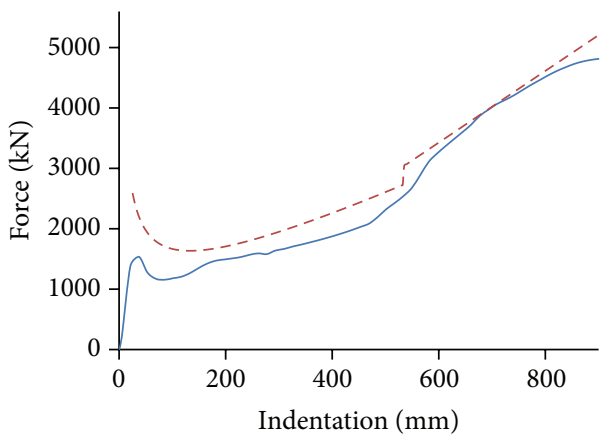

C7

- . - New method

(g)

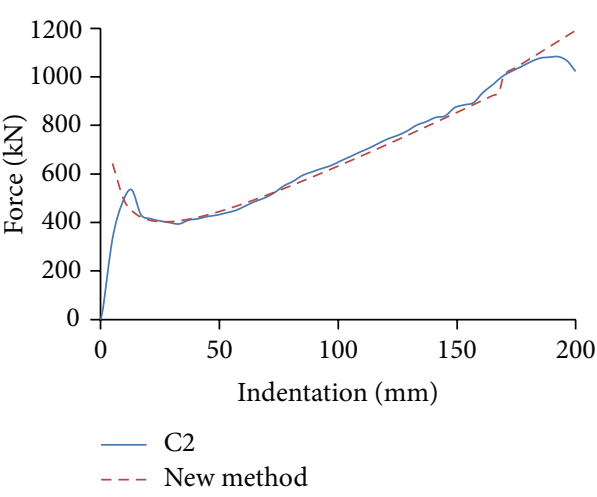

(b)

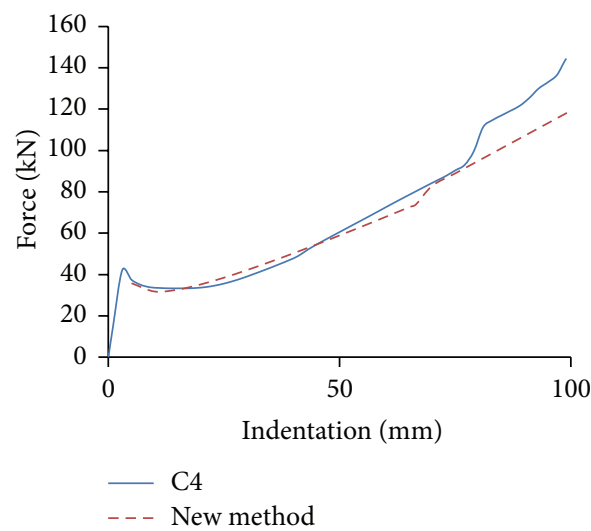

(d)

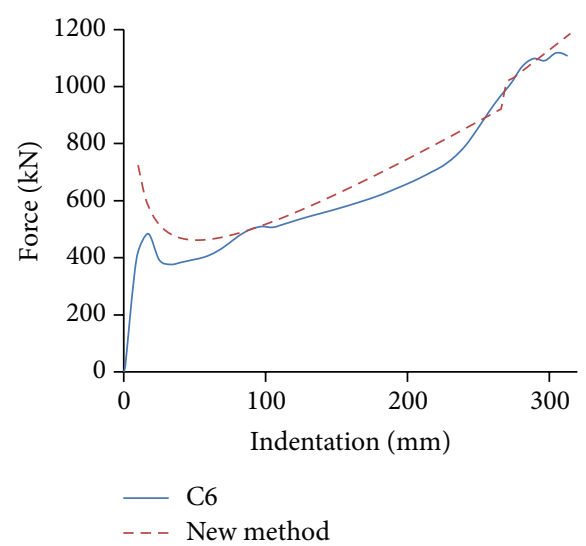

(f)

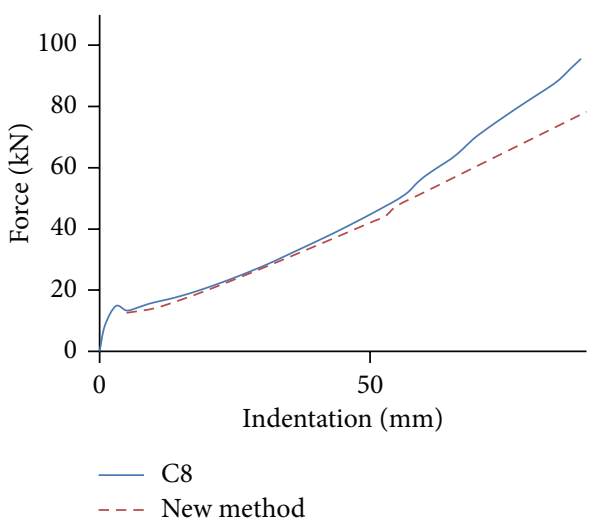

(h)

Figure 13: Continued. 


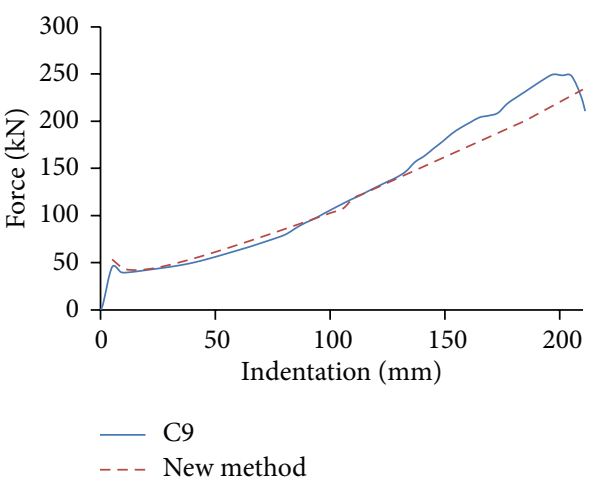

(i)

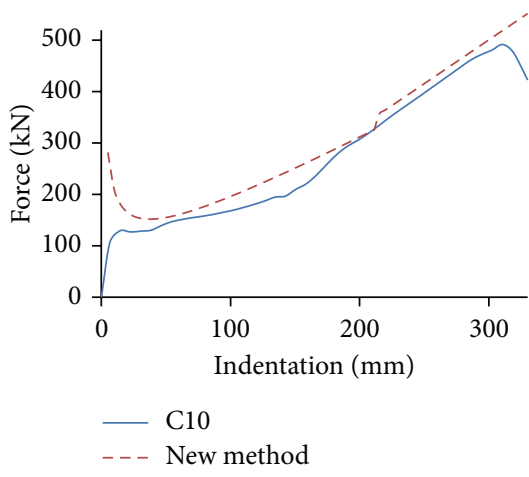

(j)

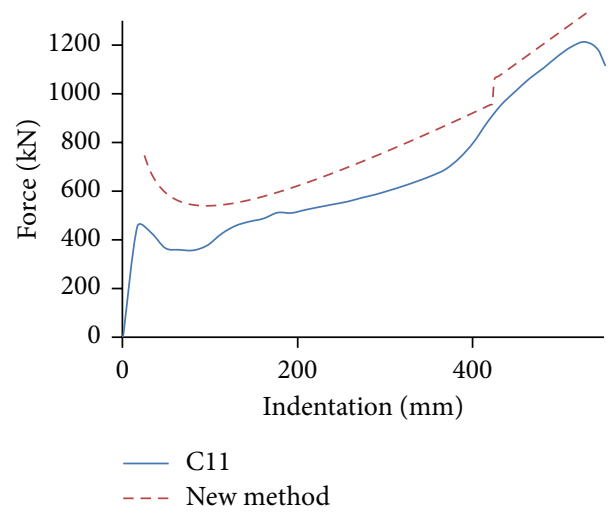

$(\mathrm{k})$

FIGURE 13: Comparisons between numerical simulations and the new method.

that the force-indentation curves obtained from the new method match well with those of experiments, except for the first peak due to the elastic buckling of the plate. Before the first peak, the plate stays in the elastic deformation stage, and the crushing resistance increases linearly. For this stage, the simplified analytical methods, which are obtained by upper bound theorem of plastic limit analysis, are not suitable to be used, as the energy dissipation will be significantly overestimated by the analytical methods, and resistance will be also dramatically overestimated, especially for the initial elastic deformation. For the subsequent folding, a little underestimation of the resistance is observed for the new method, which also occurs for other existing simplified analytical methods. This is mainly because the assumptions made during the derivation of resistance for the first fold may cause larger deviation for the subsequent folding as the geometric relationship becomes more complicated. Nonetheless, not so many folds will form in the realistic cases due to the fact that the rupture occurs; (25) can still be used for an approximation to the crushing resistance of a web girder for the subsequent folds.

Compared with other existing simplified analytical methods, the new method can give a prediction of crushing resistance which is a little smaller than that by Simonsen and Ocakli [15] but a little bigger than that by Hong and Amdahl [16]; see Figure 11.
When comparing with numerical simulations, the new method gives satisfactory prediction results in most cases, though little discrepancy does exist in some cases.

The cases of numerical simulations can be separated into three groups according to different $2 b / t$ ratios. The web girders in every group have a wide range of scantlings. By investigating the comparison results in every group, it can be found that as the size of the plate grows large, the new method may give an overestimation of the crushing resistance when the $2 b / t$ ratio is a constant. For example, in group $\mathrm{C} 4-\mathrm{C} 7$, the size of the web girder grows larger from case $\mathrm{C} 4$ to case $\mathrm{C}$. In cases $\mathrm{C} 4$ and $\mathrm{C} 5$, the resistance obtained by the new method agrees perfectly with that from numerical simulations, while in case C6, a little overestimation is observed and in case C7 larger overestimation occurs. The similar tendency can be observed in group $\mathrm{C} 1-\mathrm{C} 3$ and group $\mathrm{C} 8-\mathrm{C} 11$.

From another view point, the eleven numerical simulation cases can be divided into four groups with four different sizes of web girders, which are $400 \mathrm{~mm} \times 200 \mathrm{~mm}, 800 \mathrm{~mm} \times$ $400 \mathrm{~mm}, 1600 \mathrm{~mm} \times 800 \mathrm{~mm}$, and $3200 \mathrm{~mm} \times 1600 \mathrm{~mm}$. Investigating the comparison results and the different $2 b / t$ ratios in each group, some conclusions can also be made. For the groups $400 \mathrm{~mm} \times 200 \mathrm{~mm}$ and $800 \mathrm{~mm} \times 400 \mathrm{~mm}$, the new method can give good predictions for all three $2 b / t$ ratios. While in the groups $1600 \mathrm{~mm} \times 800 \mathrm{~mm}$ and $3200 \mathrm{~mm} \times$ $1600 \mathrm{~mm}$, the larger $2 b / t$ ratio is, the more overestimation of 
the crushing resistance is obtained by the new method. As the length $2 b$ is the same for all cases in one group, the variation of the $2 b / t$ ratio represents the change of the plate thickness. Thus, it indicates that, for large scale plate, the thinner the web girder is, the more the overestimation is for the crushing resistance with the new method.

In summary, the accuracy of the proposed simplified analytical method for predicting the crushing resistance is satisfactory, especially for web girders with small or medium scale. For web girders with large scale, the new method may give a little overestimation of the crushing resistance, and the overestimation may be more for large scale web girders with small thickness.

\section{Conclusions}

The purpose of the present paper is to present the investigation by numerical simulations and theory on the behavior of web girders subjected to in-plane concentrated crushing load. A new theoretical deformation model was proposed, which captures the important features that were ignored by the existing theoretical deformation models. By applying the simplified plastic analytical method originating from upper bound theorem to the new theoretical deformation model, the solutions for instantaneous and mean crushing resistance of web girders are obtained for general case of an eccentric in-plane localized impact load.

The proposed simplified analytical method for crushing resistance of web girders is verified by two previous experiments and a series of numerical simulations. Through the comparisons, it can be found that the new method can give satisfactory prediction of crushing resistance for web girders with small or medium scale. For web girders with large scale, a little overestimation may occur, and small thickness may aggravate the overestimation for large web girders. The new simplified analytical method can be a useful part of fast and reliable assessment tools for the ship structure performance in accidental collision and grounding events.

\section{Nomenclature}

$a$ : Half width of the web flange plate

$b$ : Half span of the web girder

$b_{1}$ : One side of the web girder

$b_{2}$ : The other side of the web girder, $b_{2}=2 b-b_{1}$

$E_{b}$ : Bending energy dissipation

$E_{m}: \quad$ Membrane energy dissipation

$\dot{E}_{b}$ : Rate of bending energy dissipation

$\dot{E}_{\text {int }}: \quad$ Rate of internal energy dissipation

$\dot{E}_{m}$ : Rate of membrane energy dissipation

$F(\delta)$ : The instantaneous crushing resistance of the web girder

$F_{f}(\delta)$ : Resistance from the web flange plate

$F_{m}$ : Mean resistance of the web girder

$F_{w}(\delta)$ : The instantaneous crushing resistance of the web girder with attached flange
$H$ : Characteristic crushing depth of the web girder

$M_{0}$ : Fully plastic bending moment per unit length of the plate

$N_{0}$ : Fully plastic membrane force per unit length of the plate

$t$ : Plate thickness of the web girder

$t_{f}$ : Plate thickness of the web flange

$\alpha$ : Instantaneous rotation angle of the plate

$\dot{\alpha}: \quad$ Angular velocity of the hinge bending

$\delta: \quad$ Instantaneous indentation

$\dot{\delta}$ : Crushing velocity

$\sigma_{y}$ : Yield stress of the material

$\sigma_{u}$ : Ultimate stress of the material

$\sigma_{0}:$ Flow stress, $\sigma_{0}=\left(\sigma_{y}+\sigma_{u}\right) / 2$

$\dot{\varepsilon}_{\text {avg }}$ : Average tensile strain rate in the length direction of the deformed plate

$\lambda$ : $\quad$ Effective crushing factor.

\section{Conflict of Interests}

The authors declare that there is no conflict of interests regarding the publication of this paper.

\section{References}

[1] S. Haris and J. Amdahl, "Analysis of ship-ship collision damage accounting for bow and side deformation interaction," Marine Structures, vol. 32, pp. 18-48, 2013.

[2] Z. Yu, Z. Hu, J. Amdahl, and Y. Liu, "Investigation on structural performance predictions of double-bottom tankers during shoal grounding accidents," Marine Structures, vol. 33, pp. 188213, 2013.

[3] H. Zhiqiang, A. Jørgen, and H. Lin, "Verification of a simplified analytical method for predictions of ship groundings over large contact surfaces by numerical simulations," Marine Structures, vol. 24, no. 4, pp. 436-458, 2011.

[4] O. Kitamura, "FEM approach to the simulation of collision and grounding damage," Marine Structures, vol. 15, no. 4-5, pp. 403428, 2002.

[5] H. Endo, Y. Yamada, O. Kitamura, and K. Suzuki, "Model test on the collapse strength of the buffer bow structures," Marine Structures, vol. 15, no. 4-5, pp. 365-381, 2002.

[6] Y. Yamada and H. Endo, "Experimental and numerical study on the collapse strength of the bulbous bow structure in oblique collision," Marine Technology, vol. 45, no. 1, pp. 42-53, 2008.

[7] H. S. Alsos, J. Amdahl, and O. S. Hopperstad, "On the resistance to penetration of stiffened plates, part II: numerical analysis," International Journal of Impact Engineering, vol. 36, no. 7, pp. 875-887, 2009.

[8] J. R. Amdahl, "Energy absorption in ship-platform impacts," Tech. Rep. UR-83-34, Division of Marine Structures, University of Trondheim, Trondheim, Norway, 1983.

[9] G. Wang, Structural analysis of ships' collision and grounding [Ph.D. thesis], Department of Naval Architecture and Ocean Engineering, University of Tokyo, Tokyo, Japan, 1995.

[10] B. C. Simonsen, Mechanics of ship grounding [Ph.D. thesis], Technical University of Denmark, 1997. 
[11] L. Hong, Simplified analysis and design of ships subjected to collision and grounding [Ph.D. dissertation], Norwegian University of Science and Technology, 2009.

[12] T. Wierzbicki and J. Culbertson Driscoll, "Crushing damage of web girders under localized static loads," Journal of Constructional Steel Research, vol. 33, no. 3, pp. 199-235, 1995.

[13] B. C. Simonsen, "Ship grounding on rock, I: theory," Marine Structures, vol. 10, no. 7, pp. 519-562, 1997.

[14] S. Zhang, The mechanics of ship collisions [Ph.D. thesis], Technical University of Denmark, 1999.

[15] B. C. Simonsen and H. Ocakli, "Experiments and theory on deck and girder crushing," Thin-Walled Structures, vol. 34, no. 3, pp. 195-216, 1999.

[16] L. Hong and J. R. Amdahl, "Crushing resistance of web girders in ship collision and grounding," Marine Structures, vol. 21, no. 4, pp. 374-401, 2008.

[17] L. Hong and J. R. Amdahl, "Plastic mechanism analysis of the resistance of ship longitudinal girders in grounding and collision," Ships and Offshore Structures, vol. 3, no. 3, pp. 159171,2008 .

[18] T. Kuroiwa, "Dynamic experiments and numerical simulations of failure of ships side structure," in Procedings of the Conference on Prediction Methodology of Tanker Structural Failure and Consequential Oil Spill, 1993.

[19] J. O. Hallquist, LS-DYNA: Theoretical Manual, Livermore Software Technology Corporation, Livermore, Calif, USA, 1993.

[20] M. S. Samuelides, G. Voudouris, M. Toulios, J. R. Amdahl, and R. Dow, "Simulation of the behaviour of double bottoms subjected to grounding actions," in Proceedings of International Conference for Collision and Groundings, 2007.

[21] W. Abramowicz, "The effective crushing distance in axially compressed thin-walled metal columns," International Journal of Impact Engineering, vol. 1, no. 3, pp. 309-317, 1983. 


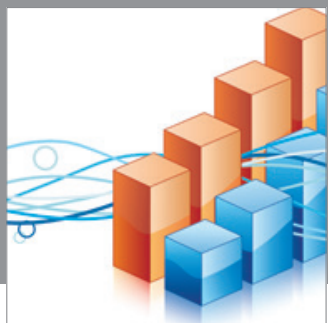

Advances in

Operations Research

mansans

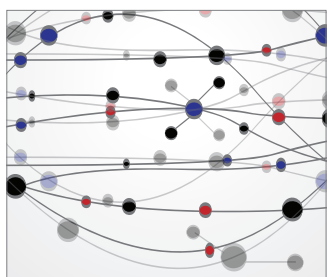

The Scientific World Journal
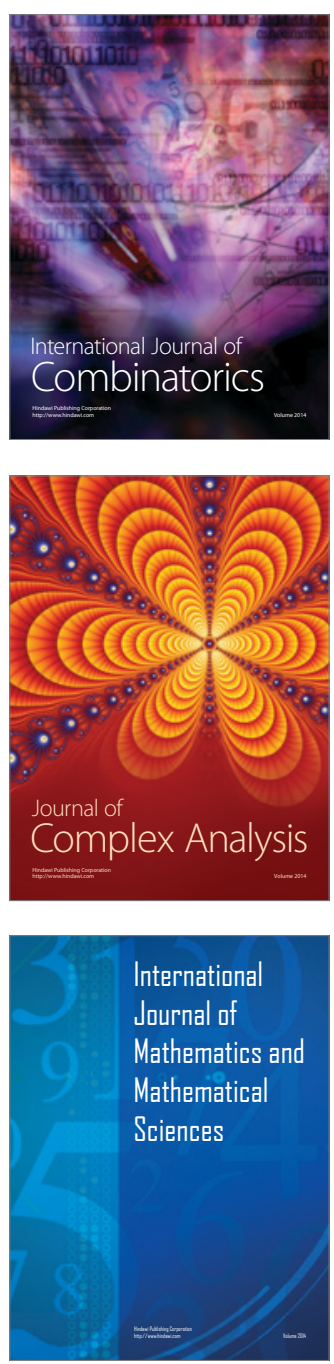
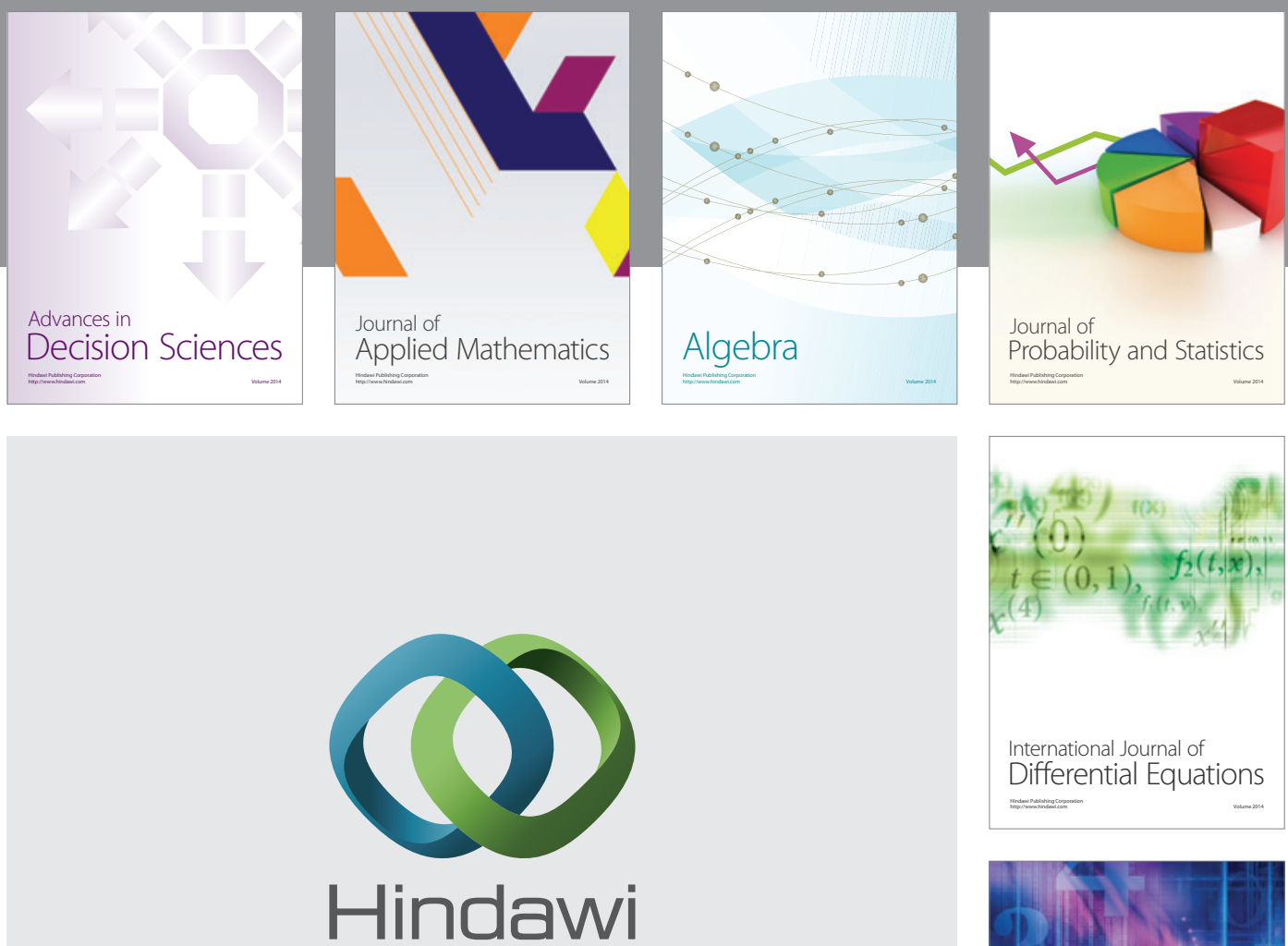

Submit your manuscripts at http://www.hindawi.com
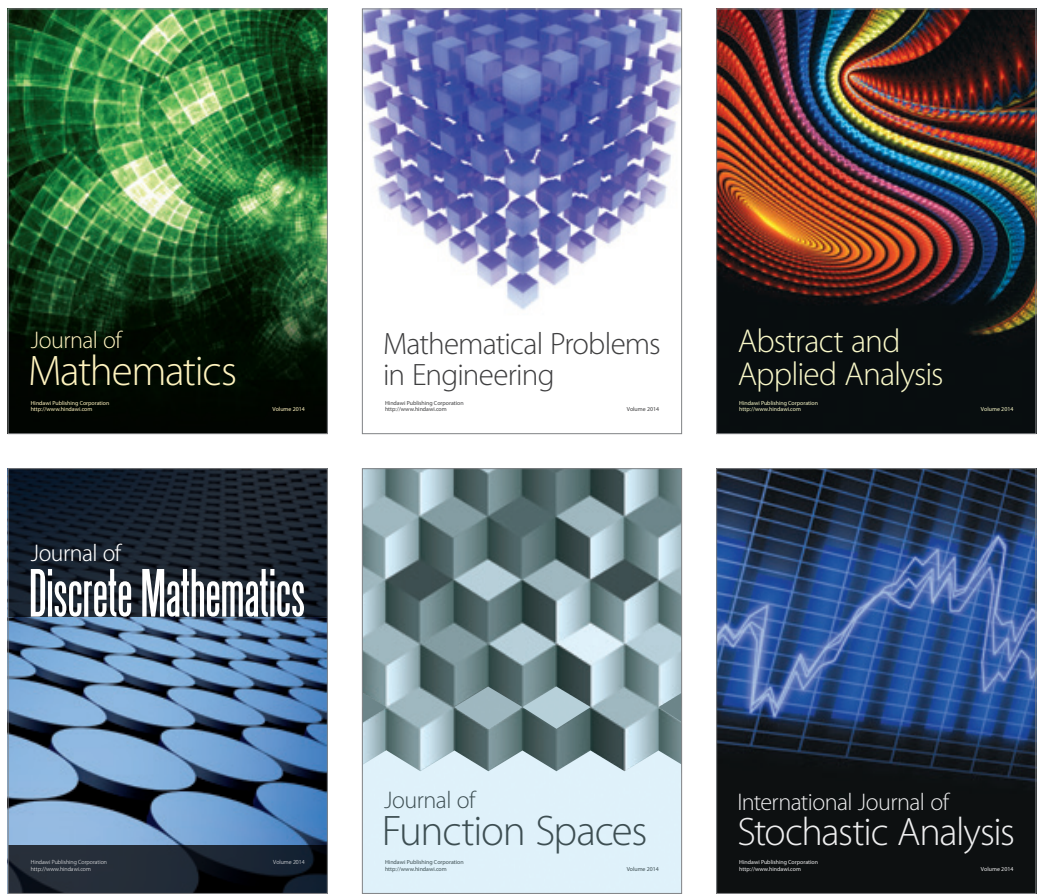

Journal of

Function Spaces

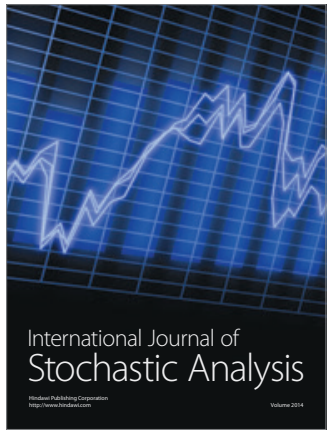

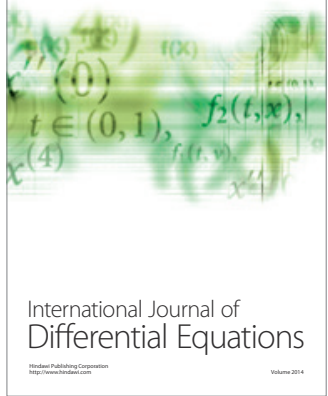
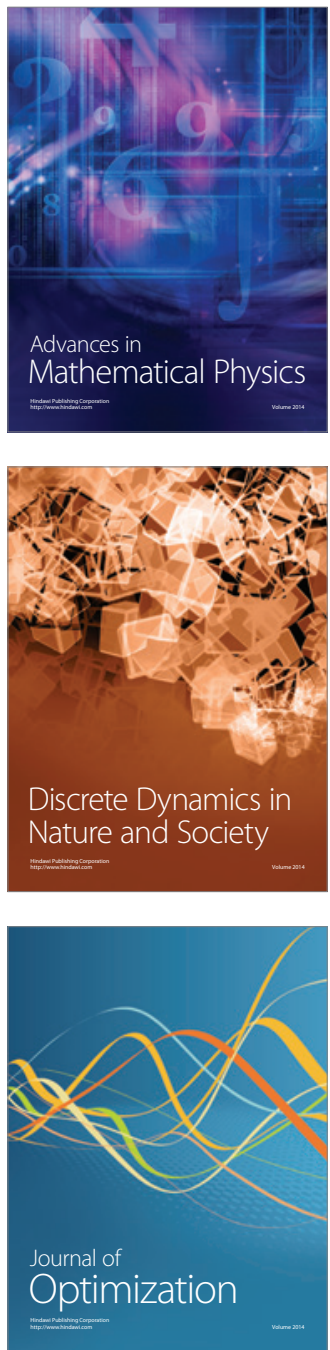